\title{
Incidence and characteristics of heterotopic ossification after spinal cord injury: a single institution study in India
}

\author{
Nidhi Rawat ${ }^{1} \cdot$ Sushil Chugh ${ }^{1} \cdot$ Kurian Zachariah ${ }^{1} \cdot$ Santu Ghosh $^{2}$ \\ Received: 26 April 2019 / Revised: 22 July 2019 / Accepted: 25 July 2019 \\ (c) The Author(s), under exclusive licence to International Spinal Cord Society 2019
}

\begin{abstract}
Study design This was a single-centre, retrospective, descriptive, hospital-based study in persons with spinal cord injuries (SCI) patients.

Objectives To study the incidence and characteristics of heterotopic ossification (HO) after SCI.

Setting The in-patient services of the Department of Physical Medicine and Rehabilitation of a tertiary care institute in India between January 2001 and December 2017.

Methods Medical records of all consecutive patients with diagnosis of SCI in the age group of 15-60 years were reviewed for presence of HO (diagnosed by clinical signs, laboratory investigations (ALP, ESR and X-rays)) and characteristics of HO. R-Ver 3.4.2 was used for analysis and correlations. Results were considered significant at $P<0.05$.

Results A total of 303 patients satisfied inclusion criteria. Nineteen individuals $(6.3 \%)$ had developed HO. Seven $(37 \%)$ were diagnosed within 3 months of SCI. Twelve $(63 \%)$ patients developed unilateral HO. The most common site for HO was hip joint $(73 \%)$. A significant association was found between the presence of a pressure ulcer and development of $\mathrm{HO}$ $(P=0.01)$.

Conclusions The incidence of $\mathrm{HO}$ was $6.3 \%$ in our institution and the hip joint is the most common site. Due to the presence of limited treatment options it is important to diagnose HO early in patients with SCI based on clinical features and later confirmed with laboratory tests and imaging.
\end{abstract}

\section{Introduction}

Heterotopic ossification (HO) is the formation of lamellar bone within the soft tissue surrounding a joint. The incidence following a spinal cord injury (SCI) ranges between 1 and $50 \%$ which depends upon multiple factors like the study design and methods used for diagnosing $\mathrm{HO}$ [1]. HO is usually found in the first 6 months post SCI with a peak at 2 months after SCI [2]. About 20\% of SCI patients develop

These authors contributed equally: Sushil Chugh, Kurian Zachariah, Santu Ghosh

Nidhi Rawat

dr.nidhi_rawat@yahoo.co.in

1 Department of Physical Medicine \& Rehabilitation, St. John's Medical College Hospital, Bengaluru, Karnataka, India

2 Department of Biostatistics, St. John's Medical College Hospital, Bengaluru, Karnataka, India clinically significant $\mathrm{HO}[2,3]$. Ankylosis of joint, pressure ulcers, nerve entrapment, deep vein thrombosis (DVT) and pain are the associated complications [3]. The clinical presentation is quite varied from an incidental $\mathrm{X}$-ray finding to severe limitation of joint range of motion (ROM) [2]. Both hereditary and acquired factors can contribute to the pathogenesis of HO [4]. During the acute phase, HO presents like an acute inflammatory condition with localised erythema, swelling, warmth, and restriction of joint motion. Risk factors for $\mathrm{HO}$ include older age (children and adolescents have a lower incidence), neurological complete lesions, male gender, spasticity, DVT and pressure sores [1-8]. HO is found to occur below the level of the SCI and the most common site being the hip (70-97\%) [2-6]. There is no specific diagnostic criteria for $\mathrm{HO}$, and it is extremely difficult to predict which spinal injury patient would subsequently develop HO [6]. HO is primarily diagnosed based on clinical signs, raised serum alkaline phosphatase (SAP) levels with positive imaging findings. The most sensitive technique for diagnosing $\mathrm{HO}$ is three-phase bone scan. CT and MRI can also aid in diagnosis but usually done if 
surgical interventions are planned [2]. During the acute inflammatory phase treatment consists of providing rest to the affected limb and indomethacin and bisphosphonates can be prescribed till acute inflammation has subsided with return of erythrocyte sedimentation rate (ESR) and SAP levels to normal [4]. Low-field irradiation and surgical resection are other options for management of HO [2]. In patients where $\mathrm{HO}$ interferes with functional abilities of an individual one can plan surgical excision of HO. Surgery when combined with radiation therapy helps in preventing postoperative recurrence [3]. Effect of prophylactic treatment with nonsteroidal anti-inflammatory drugs or radiation therapy to prevent $\mathrm{HO}$ has not been studied in India. Treatment of $\mathrm{HO}$ in India most commonly consists of indomethacin, disodium etidronate and physical therapy and less commonly radiation therapy and surgery. Radiation and surgery are restricted to individuals with $\mathrm{HO}$ causing severe restriction in the functional abilities and activities of daily living. The response to treatment is limited once $\mathrm{HO}$ is completely formed. Despite widespread knowledge about HO in SCI there have not been any reports assessing the development of $\mathrm{HO}$ in India. The current study will help in elucidating incidence and characteristics of $\mathrm{HO}$ and potential risk factors in developing $\mathrm{HO}$ following SCI.

\section{Methods}

This was a single-centre, retrospective, descriptive, hospital-based study in SCI patients who attended the inpatient services of the Department of Physical Medicine and Rehabilitation of a tertiary care institute in India between January 2001 and December 2017. The Institute Ethics Committee approval was sought before initiation of the study. Medical records of all consecutive patients diagnosed with SCI aged 15-60 years were reviewed for presence or absence of HO. Clinical signs to diagnose $\mathrm{HO}$ were fever, erythema, warmth, swelling and limitation in joint ROM. Blood investigations like erythrocyte sedimentation rate (ESR) and SAP levels were noted. We also reviewed Xrays, computerised axial tomography scans, ultrasonography and bone scans. X-ray findings suggestive of $\mathrm{HO}$ varied from an increased density of the peri-articular soft tissues to mature extra-osseous cortical and trabecular new bone formation. Patients with a premorbid history of $\mathrm{HO}$, long bone fracture and head injury were excluded from the study. Age, gender, time since injury, level and degree of SCI and the presence of DVT, urinary tract infection (UTI) and pressure ulcers was recorded. Treatment charts of patients previously identified with $\mathrm{HO}$ were also reviewed. All study participants with $\mathrm{HO}$ were treated with indomethacin for 2-3 weeks. All study individuals with HO continued to receive ROM exercises. Vigorous exercises and forceful stretching were stopped till acute inflammation subsided. The demographic and clinical parameters were summarised by frequency and percentages and were compared based upon the presence or absence of HO. Chisquare test was used to determine statistical significance at $P<0.05$. Data were analysed using the statistical software R-Ver 3.4.2 (R Core Team, 2018) [9].

\section{Results}

Three hundred and three patients who regularly followed at our institution satisfied the inclusion criteria. Nineteen patients $(6.3 \%)$ had developed $\mathrm{HO}$ and all were diagnosed at our centre. Seven $(37 \%)$ were diagnosed within 3 months of SCI. Four were diagnosed with $\mathrm{HO}$ between 3 and 6 months, one between 7 and 12 months and the rest seven after 12 months of SCI. Amongst those who developed HO, 14 were $<35$ years. Seventeen were males. All individuals who developed HO sustained traumatic SCI. The neurological level of injury was cervical in eight (42.1\%), upper thoracic in two (10.5\%), lower thoracic in nine (47.3\%) patients. Seventeen $(89 \%)$ patients who developed HO were AIS A (American Spinal Injury Association (ASIA) Impairment Scale) meaning complete SCI. Eight (42.1\%) patients who developed HO had tetraplegia and the rest 11 had paraplegia. Twelve (63\%) patients developed unilateral HO. The most common site for HO was hip joint $(73 \%)$ (Left $>$ right) followed by knee joint (42\%). A total of 50\% of patients developed $\mathrm{HO}$ at more than one site.

Table 1 summarises the demographics and clinical correlates of HO in study patients. Pressure ulcers were found in $16(84.2 \%)$ SCI patients who developed HO. Significant association was found between presence of pressure ulcer and development of HO $(P=0.01)$. There were no significant associations with UTI or DVT.

\section{Discussion}

$\mathrm{HO}$ is a well-known complication following SCI. The incidence of $\mathrm{HO}$ varies between $1 \%$ and $50 \%$ after SCI [1]. However, the incidence of $\mathrm{HO}$ after SCI is still unknown in India. We carried out a study to find the incidence of $\mathrm{HO}$ in SCI individuals in a cohort of people from India and identify risk factors associated with its occurrence. Nineteen patients $(6.3 \%)$ were found to develop HO. The incidence of $\mathrm{HO}$ in our cohort from India is lower than the incidence found in most other studies.

Wittenberg et al. [1] carried out a study on 356 SCI patients and concluded that $\mathrm{HO}$ was found more often in male patients $(23 \%)$ than in female $(10 \%)$ and was mostly seen between 20 and 30 years. In our study HO was more common 
Table 1 Clinical correlates of heterotopic ossification (HO)

\begin{tabular}{|c|c|c|c|c|}
\hline Variable & Components & $\begin{array}{l}\mathrm{HO} \\
\text { present }\end{array}$ & HO absent & $p$ value \\
\hline \multirow[t]{4}{*}{ Age } & $15-25$ years & 4 & 86 & 0.3 \\
\hline & 26-34 years & 10 & 90 & \\
\hline & $35-50$ years & 4 & 82 & \\
\hline & $51-60$ years & 1 & 26 & \\
\hline \multirow[t]{2}{*}{ Sex } & Male & 17 & 251 & 1 \\
\hline & Female & 2 & 33 & \\
\hline \multirow[t]{2}{*}{ Type of Injury } & Complete & 17 & 179 & 0.05 \\
\hline & Incomplete & 2 & 105 & \\
\hline \multirow[t]{4}{*}{ Time since injury } & $<3$ months & 7 & 186 & 0.07 \\
\hline & 3-6 months & 4 & 39 & \\
\hline & $7-12$ months & 1 & 29 & \\
\hline & $>12$ months & 7 & 30 & \\
\hline \multirow[t]{4}{*}{ NLI } & Cervical & 8 & 74 & 0.11 \\
\hline & Upper thoracic & 2 & 44 & \\
\hline & Lower thoracic & 9 & 105 & \\
\hline & Lumbar & 0 & 61 & \\
\hline \multirow[t]{2}{*}{ DVT } & Present & 1 & 12 & 1 \\
\hline & Absent & 18 & 272 & \\
\hline \multirow[t]{2}{*}{ UTI } & Present & 6 & 60 & 0.4 \\
\hline & Absent & 13 & 224 & \\
\hline \multirow[t]{2}{*}{ Pressure ulcer } & Present & 16 & 145 & 0.01 \\
\hline & Absent & 3 & 139 & \\
\hline
\end{tabular}

in males and mostly seen between 25 and 35 years of age. However, these results were not statistically significant.

$\mathrm{HO}$ was mostly seen after injuries of the lower cervical or thoracic spine with thoracic trauma being the most common level of injury [1]. Cervical and lower thoracic was the most common level of injury in our study cohort. Eleven out of nineteen study patients who developed HO had paraplegia. There exists no clear association between $\mathrm{HO}$ and level of SCI [6, 7]. However, Erhan et al. [3] found HO was more frequent in paraplegics.

All study individuals who developed $\mathrm{HO}$ had traumatic SCI. This could be due to the reason that our study included mostly traumatic SCI (90.7\%). Previous studies have reported a lower incidence of HO between 6 and $15 \%$ in non-traumatic myelopathies [2].

The time at which $\mathrm{HO}$ occurs after $\mathrm{SCI}$ is variable. In seven of these nineteen individuals $\mathrm{HO}$ was diagnosed within 3 months of SCI. Our study results are similar to a study done by Bravo-Payno et al. [6] on 654 SCI patients where the mean time since injury was 40.79 days. Van Kuijk et al. [2] also found that $\mathrm{HO}$ presents within the first 3 weeks after SCI.

We found significant association between the presence of pressure ulcer and $\mathrm{HO}$ development $(P$ value $=0.01)$. This supports earlier studies which found a significant association between pressure ulcer and $\mathrm{HO}$ formation $[2,4,6]$. Inflammation is known to play a role in $\mathrm{HO}$ and pressure ulcers would represent an area of increased inflammation. Pressure sores may be seen as a late complication of HO, but since they occur more commonly before the clinical onset of $\mathrm{HO}$, they could be considered a risk factor $[2,7]$. However, there are studies to show that pressure ulcers do not influence HO development [7, 8]. Hence, it is difficult to draw a definitive conclusion about association between pressure ulcers and HO. We could not get accurate data regarding the grading and number of pressure ulcers to enable us to study the association between the severity of pressure ulcers and presence of $\mathrm{HO}$.

Most studies found a significant correlation between completeness of injury and HO formation [6-8].

Our results can be considered as close to being statistically significant $(P$ value $=0.05)$. We did not find any significant correlation between development of $\mathrm{HO}$ and occurrence of UTI and DVT.

The most common site for HO formation was hip joint. Ten patients had $\mathrm{HO}$ formation at more than one site. The most common site of $\mathrm{HO}$ formation in other similar studies was also the hip joint [2-6]. Bravo-Payno et al. [6] found 24 out of $44 \mathrm{HO}$ to occur at one site. Ohlmeier et al. [10] found that $\mathrm{HO}$ most commonly occurs in the gluteal muscle group. They also concluded that since there is unavailability of specific tests for screening of $\mathrm{HO}$, routine ultrasound screening particularly of the gluteal muscles can help in detecting early HO. Exercises focussing on hip extension and external rotation can be done after SCI to prevent HO. However, in a study done by Erhan et al. [3] the most common site of HO was at knee joints followed by hip joints.

We need to conduct similar studies in other centres across India to find the overall incidence of $\mathrm{HO}$ in India.

\section{Limitation of the study}

One limitation of our study is that all patients who did not have clinical evidence of $\mathrm{HO}$ were not screened for $\mathrm{HO}$ so we might have included some $\mathrm{HO}$ cases in the nonHO group.

\section{Conclusion}

The incidence of $\mathrm{HO}$ was $6.3 \%$ in our institution and the hip joint is the most common site. Due to presence of limited treatment options it is important to diagnose $\mathrm{HO}$ early in patients with SCI based on clinical features and later confirmed with laboratory tests and imaging. 


\section{Compliance with ethical standards}

Conflict of interest The authors declare that they have no conflict of interest.

Publisher's note: Springer Nature remains neutral with regard to jurisdictional claims in published maps and institutional affiliations.

\section{References}

1. Wittenberg RH, Peschke U, Bötel U. Heterotopic ossification after spinal cord injury. Epidemiology and risk factors. J Bone Jt Surg Br. 1992;74:215-8.

2. Van Kuijk AA, Geurts AC, van Kuppevelt HJ. Neurogenic heterotopic ossification in spinal cord injury. Spinal Cord. 2002;40:313-26. https://doi.org/10.1038/sj.sc.3101309.

3. Erhan B, Gunduz B, Vural M, Yalcinkaya EY, Kahraman F, Satir O. The incidence and characteristics of heterotopic ossification in patients with spinal cord injury. SM J Neurol Disord Stroke. 2016;2:1013.

4. Taly AB, Nair KP, Jayakumar PN, Ravishankar D, Kalaivani PL, Indiradevi $\mathrm{B}$, et al. Neurogenic heterotopic ossification: a diagnostic and therapeutic challenge in neurorehabilitation. Neurol India. 2001;49:37-40.

5. Hassard GH. Heterotopic bone formation about the hip and unilateral decubitus ulcers in spinal cord injury. Arch Phys Med Rehabil. 1975;56:355. \pm 358 .

6. Bravo-Payno P, Esclarin A, Arzoz T, Arroyo O, Labarta C. Incidence and risk factors in the appearance of heterotopic ossification in spinal cord injury. Paraplegia. 1992;30:740-5.

7. Lal S, Hamilton BB, Heinemann A, Betts HB. Risk factors for heterotopic ossification in spinal cord injury. Arch Phys Med Rehabil. 1989;70:387-90.

8. Citak M, Suero EM, Backhaus M, Aach M, Godry H, Meindl R, et al. Risk factors for heterotopic ossification in patients with spinal cord injury: a case control study of 264 patients. Spine. 2012;37:1953-7. https://doi.org/10.1097/BRS.0b013e31825ee81b.

9. R Core Team. R: a language and environment for statistical computing. Vienna, Austria: R Foundation for Statistical Computing; 2018. https://www.R-project.org/.

10. Ohlmeier M, Suero EM, Aach M, Meindl R, Schildhauer TA, Citak M. Muscle localization of heterotopic ossification following spinal cord injury. Spine J. 2017;17:1519-22. https://doi.org/10. 1016/j.spinee.2017.04.021. 